\title{
Applying Fuzzy Theory to Develop a Model for Inspecting and Assessing Soil and Water Conservation Facilities
}

\author{
Pan, Nai- Hsin \\ Department of Construction Engineering \\ National Yunlin University of Science and Technology \\ Yunlin, Taiwan, R.O.C. \\ E-mail: pannh@yuntech.edu.tw
}

\author{
Chen, Kuei-yen \\ Graduate School of Engineering Science and \\ Technology \\ National Yunlin University of Science and Technology \\ Yunlin, Taiwan, R.O.C. \\ E-mail: g9610817@yuntech.edu.tw
}

\begin{abstract}
The majority of soil and water conservation facilities are located in remote areas, posing measurement difficulties involving precision instruments. Therefore, visual inspection with the advantage of rapidness is the primary assessment method used. However, standards regarding identification of the degree and relevancy parameters of damage may vary among inspectors. To overcome inspector subjectivity caused by various scoring systems, this study used fuzzy set theory to build an evaluation model that defines the membership functions of the semantics range. The semantics were used to infer current facility conditions, which were classified into various condition index (CI) levels. Also, based on the analysis results of condition index (CI) by the proposed model, an overall structural-functional index was used to obtain parameter $U$, which indicates the maintenance urgency for facilities in specific regions. The results could serve as a reference for management to formulate decisions regarding facility maintenance. This study used a systematic assessment method to reduce differences caused by inspector subjectivity and enhance assessment consistency of current structural conditions and maintenance urgency.
\end{abstract}

Keywords-Fuzzy, inspection, maintenance, assessment, Infrastructur

\section{INTRODUCTION}

From a life-cycle perspective, the construction cost accounts for a small proportion of the life of a structure, in which $60 \%$ of the life-cycle cost is consumed by regular checks of the structures and maintenance. However, the inspection methods and assessment criteria used to evaluate soil and water conservation facilities in Taiwan are inconsistent and thus cannot be used as references for decision supporting. Visual inspection is used extensively in Taiwan as a method for examining and maintaining public works such as bridges, tunnels, and harbors. However, because of differences in locations and functions of public structures, different inspection criterias must be defined. The visual inspection is currently one of the most efficient and convenient methods to inspect a large number of structures that are widely distributed, and thus is primarily used in this study for examining soil and water conservation structures. The visual inspection and assessment results of facilities indicate that inspectors adopt differing criteria for determining the extent of damage and that human factors cause inconsistent scores during the scoring process.

To reduce inconsistencies in inspection results, this study was to resolve the current matters existing in the inspection. Nevertheless, the main reason of the assessment criteria differences used by various units is differing subjective semantics. One of possible alternatives to solve this problem is to apply fuzzy set theory for reducing semantical differences. In this study, a semantic scale that was developed by applying fuzzy set theory was employed to convert and reduce the scoring problems in the visual inspection. Based on the extent of damage and functional impact of the damaged structures, an assessment scale was developed that could be used by soil and water conservation departments. In addition, the scope of the membership function was defined, and fuzzy inference were undertaken to obtain a condition index (CI) score of the overall structure, which could be used to assess the functionality of structures in particular regions.

Thus, this study employed fuzzy set theory to integrate the assessment scores, thereby providing actual modified inspection scores for the assessment. Systematically evaluating the conditions of the structures and defining the scope of the extent and effects of damage are beneficial for understanding the condition indices of the structures and assessing the extent of damage to the structures. Subsequently, the functional indicators of the overall structures can be integrated with the maintenance urgency to indicate the urgency of required maintenance in particular areas. This method enables effective recommendations and facilitates quick responses, thereby achieving effective improvement on torrent inspection and management. In addition, the urgent maintenance assessments can be ranked to provide a reference for maintenance units for prioritizing maintenance work. The purpose of this study was to use fuzzy inference to resolve visual inspection problems, which are primarily the differences resulting from the semantic cognition that inspectors develop during assessments. The CI and the CIlevel conversion yielded by defuzzification using fuzzy inference can be adopted to understand the CI-level of the structures, thereby enhancing the assessment consistency. Finally, the overall structural function index and the CI 
were used to calculate the level of urgency systematically. The rankings of the urgency values can serve as references for maintenance units. This study are focused on soil and water conservation facilities that were installed underwater and have been existed and in operation for over 5 years, including groundsill works, bank revetments, and sabo dams.

\section{LITERATURE REVIEW}

Water conservation structures may contain defects. Several defects are caused by impacts such as current scour or debris flow, but other defects may be caused by improper design, construction, or maintenance. Bridge management in Taiwan relies on visual inspections that assess degree, extent, relevancy, and urgency (DER\&U) parameters. This visual inspection and assessment was developed by the Taiwan Area National Freeway Bureau (TANFB) and serves as guidelines for bridges. Poveda et al. developed a fuzzy logic model for predicting and evaluating the work performance of construction foremen. The primary purpose of this model is to assess the effectiveness of foremen by monitoring their improvement over time and identifying areas in which foremen require training to improve their performance [2]. Reshmidevi et al. performed suitability evaluations in arid agricultural watersheds to assess the potential for supplementary irrigation in surrounding areas. Because of uncertainty and vagueness, fuzzy sets were used as the basis of the research. Decision-making criteria were converted to fuzzy sets to assess land suitability based on the environment and theconsideration of both land potential and surface water potential [3]. Liu and Wang explored third-party logistics (3PL) and discovered supplier demands had become increasingly significant. Therefore, to improve customer service and reduce costs, these scholarsproposed an integrated fuzzy method for assessing and selecting 3PL providers and applying fuzzy inference methods to eliminate unacceptable providers [1] Saleh and Kim proposed a fuzzy system for evaluating student grades. The proposed system uses fuzzification, fuzzy inference, and defuzzification based on difficulty considerations to resolve criticalcomplex questions. They suggested that because of the transparency, objectivity, and ease of applying the system to automatically evaluate student grades, this system is more reasonable and fair [4]. Nahid Rezaeiniya (2012), describe the research and development of hybrid MCDM methods for greenhouse locating. Selection of the most appropriate location for investor is an important problem which requires assessment and analysis of several factors. The paper clarifies the structure of important criteria in greenhouse locating [5].

\section{APPLYING FUZZY SET THEORY TO VISUAL INSPECTIONS}

\section{Disadvantages of visual inspection}

The Taiwan Bridge Management System has extensively applied the visual inspection method when inspecting bridges, and the parameters used for assessments have been the DER\&U. The visual inspection assessment principle was formulated by the Taiwan Area
National Freeway Bureau for the Bridge Management System. When conducting the visual inspection assessment, the convenience to the inspector should be considered and structural deterioration should be adequately expressed. Each parameter range that is not excessive or insufficient. The DER\&U assessment method is divided into four types of assessments. The advantage of visual inspection is that the work of entering information can be reduced by using numbers to concisely record the inspection results; therefore, visual inspection methods are convenient when requiring numerous and rapid structural surveys. However, the disadvantage of visual inspections is that data can easily overlap because the inspections are not coded. By coding the structures based on the extent of structural damage, the damage condition of the facilities can be highlighted for subsequent analysis and maintenance.

\section{Applying fuzzy set theory}

This study employed fuzzy semantic inference to resolve the primary problems resulting from visual inspections (i.e., the differences in the semantic judgments and quantification processes of inspectors). Therefore, judgments regarding semantics and scores were first integrated before referential semantics and scores were developed. Applying fuzzy sets by integrating expert opinions is a common method; fuzzy preference relationships can be used to obtain the intersection of expert opinions. Therefore, this study conducted interviews and adopted fuzzy set theory to redefine the membership score functions. Fuzzy set theory was used in this study primarily because fuzzy theory can be used to describe indefinite logical problems and convert semantics into reasonable intervals. This following section explains the procedure of applying fuzzy set theory.

The Mamdani-style fuzzy inference is as follows.

- Fuzzification: Linking the input values to

corresponding fuzzy memberships

- Rule evaluation: Determining the correspondence degree of the rules

- Defuzzification: Converting the integrated results into well-defined outputs

This study applied trapezoidal fuzzy numbers, which are common in fuzzy theory.

\section{1) Fuzzification}

This study adopted the DER\&U parameters, which are frequently used as the scoring method in visual inspections. The results of the interviews showed that although in practice, parameters $\mathrm{D}$ and $\mathrm{R}$ were applicable to inspections regarding soil and water conservation facilities, parameter $\mathrm{E}$ was inapplicable because it required tools for the measurement, and parameter $U$ should be considered at an overall level. A subsequent section uses quantitative formulaic calculations to explain the calculations of parameter $U$. The primary consideration of this study is whether the inspected structures were damaged; therefore, recently built structures were excluded. D denotes the degree of the structure's damage. R denotes the degree of the structure's functionality after damage happened.

An explanation of the corresponding scores obtained after the semantic conversion is as follows: fuzzy 
semantics scores of parameter $\mathrm{R}$ were defined as low, medium, and high, and those for parameter $\mathrm{R}$ were defined as small, medium, and large.

For example, the range of the set corresponding to medium damage was between 0.2 and 0.8 . The range of the overall fuzzy set, following systematic conversion, was the membership function table for parameters D and $\mathrm{R}$, as shown in Table 1. The data were entered into Matlab after processing.

Table.1 Input Fuzzy member function definition

\begin{tabular}{cll}
\hline \multicolumn{1}{c}{ Item } & Symbol & Range of scale \\
\hline \multirow{3}{*}{ Degree (D) } & Low & {$[0,0,0.1,0.4]$} \\
& Mod & {$[0.2,0.4,0.6,0.8]$} \\
& Bad & {$[0.6,0.8,1,1]$} \\
\hline \multirow{3}{*}{ Relevancy (R) } & Small & {$[0,0,0.1,0.4]$} \\
& Mod & {$[0.2,0.4,0.6,0.8]$} \\
& Large & {$[0.6,0.8,1,1]$} \\
\hline
\end{tabular}

*Degree : D denotes the degree of the structure's damage.

* Relevancy : R denotes the degree of the structure's functionality after damage happened.

\section{2) Fuzzy rules and inference results}

During the onsite inspections of soil and water conservation facilities, damaged components are assessed. In this study, scoring systems applicable to the assessment of soil and water conservations facilities were identified, and these study assessment scores will enable relevant personnel to understand the current conditions of the facility. By using inference rules, people can understand the current conditions and delineate the overall $\mathrm{CI}$ of the facilities. CI denotes the functional degree of a single facility structure, which is calculated from the parameters $\mathrm{D}$ and $\mathrm{R}$.

Fuzzification is a tool, and data can be entered into a program to be fuzzified. Following the conversion of fuzzy membership functions, the data represent sematic descriptions. The figures 4 and 5 show the inference regarding the first part of the membership functions of parameters $\mathrm{D}$ and $\mathrm{R}$.

This study converted and outputted two types of semantic variables into a single variable. The two variables were defined as $\mathrm{X} 1$ and $\mathrm{X} 2$, representing parameters $\mathrm{D}$ and $\mathrm{R}$, respectively. The corresponding semantics, that is, \{low, moderate, high\} and \{minor, moderate, substantial $\}$ were used as the output of the CI is \{normal, bad, extremely damaged\}, as shown in Figure 6. The five inference rules are presented in the following paragraphs, and the inference method used a sum set to infer the area before using the center of gravity method for defuzzification.

\section{3) Inference rules}

(1.) If ( $\mathrm{R}$ is low) and ( $\mathrm{D}$ is small) then (CI is normal)

(2.) If ( $\mathrm{R}$ is low) and ( $\mathrm{D}$ is mod) then (CI is normal)

(3.) If ( $\mathrm{R}$ is mod) and ( $\mathrm{D}$ is mod) then (CI is bad)

(4.) If ( $\mathrm{R}$ is bad) and ( $\mathrm{D}$ is mod) then (CI is bad)

(5.) If ( $\mathrm{R}$ is bad) and (D is lag) then (CI is extremely damaged)

The semantic definition of the CI was divided into three levels, which were based on the results of fuzzy inference from the membership functions of the onsitefacility CI. The target range of the membership function is between 0 and 10 points. A CI value approaching 10 indicates severe damage; Triangular distribution was used to express the definition of the ranges of normal, bad, and extremely damaged. In particular, normal was $[0,0,0.5]$, bad was $[0,0.5,1]$, and extremely damaged was $[0.5,1,1]$. In the inference results obtained using this inference method, a high score indicated severe damage, and 5 was the median. displays are shown in table2.

\begin{tabular}{lll}
\multicolumn{3}{c}{ Table.2 Output fuzzy member function definition } \\
\hline Item & Symbol & Range of scale \\
\hline \multirow{2}{*}{ Condition index (CI) } & Normal & {$[0,0.5]$} \\
& Bad & {$[0,0.5,1]$} \\
& Vary damage & {$[0.5,1]$} \\
\cline { 2 - 2 } Condition index: denotes the functional degree of a single facility \\
structure, which is calculated from the parameters D and R.
\end{tabular}

\section{4) Defuzzification}

In this study, parameter D was used to represent the extent of damage and parameter $\mathrm{R}$ represents the level of impact. The results of systematic defuzzification show that defuzzification can accurately reflect the actual CI of a structure. This assessment value can be used to achieve effective quantification, thereby reducing the errors resulting from human factors, which was the purpose of this study. The widely used center of gravity method, which was proposed in Yager (1981) as a defuzzification method, was employed as the calculation equation in this study. In addition, this study applied the widely used mean of maximum method: The membership function was divided at the premise $\alpha$ and the maximum membership, and 1 represents the number of memberships greater than or equal to the premise $\alpha$ or the defined membership, (Eq.1). \#

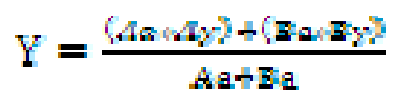

Y: Defuzzification explicit conversion value Aa,Ba: A and B Area

Ay,By: A and B the center of gravity

\section{5) Fuzzy inference verification}

The verification process of this study involved inferring results from five historical cases. The CI values obtained after the first ratings of the five cases were marked as "CI-before" and were converted according to the functional classification scale defined in this study. Users were then asked to assign scores for these five historical cases. In the returned questionnaires, "D-max" and "R-max" represented the maximal values selected for parameters $\mathrm{D}$ and $\mathrm{R}$, respectively. The minimal values selected for these parameters were represented by "Dmin" and "R-min," respectively. In addition, the results of fuzzy inference were expressed using "CI-min" and "CImax." Subsequently, the verified fuzzy inference CI values were converted to CI-levels. Finally, these results were compared with the previous ratings to determine whether they were consistent. Table 3 shows that the results were consistent, indicating that the results obtained using fuzzy inference were within a reasonable range. 
Table 3 Results of the verification obtained using fuzzy inference

\begin{tabular}{lllllllll}
\hline & $\begin{array}{l}\text { D- } \\
\text { min }\end{array}$ & $\begin{array}{l}\text { D- } \\
\text { max }\end{array}$ & $\begin{array}{l}\text { R- } \\
\mathbf{m i n}\end{array}$ & $\begin{array}{l}\text { R- } \\
\mathbf{m a x}\end{array}$ & $\begin{array}{l}\text { CI- } \\
\mathbf{m i n}\end{array}$ & $\begin{array}{l}\text { CI- } \\
\mathbf{m a x}\end{array}$ & $\begin{array}{l}\text { CI- } \\
\text { level }\end{array}$ & $\begin{array}{l}\text { CI } \\
\text { level } \\
\text { before }\end{array}$ \\
\hline 1 & 0.2 & 0.3 & 0.1 & 0.2 & 0.18 & 0.21 & $\mathrm{~A}$ & $\mathrm{~A}$ \\
\hline 2 & 0.8 & 0.9 & 0.7 & 0.8 & 0.81 & 0.86 & $\mathrm{C}$ & $\mathrm{C}$ \\
\hline 3 & 0.4 & 0.7 & 0.3 & 0.5 & 0.47 & 0.5 & $\mathrm{~B}$ & $\mathrm{~B}$ \\
\hline 4 & 0.5 & 0.8 & 0.4 & 0.6 & 0.5 & 0.5 & $\mathrm{~B}$ & $\mathrm{~B}$ \\
\hline 5 & 0.8 & 1 & 0.7 & 0.8 & 0.81 & 0.84 & $\mathrm{C}$ & $\mathrm{C}$ \\
\hline \multicolumn{8}{c}{ *Authentication, a random sample of five cases verified as a } \\
representative description
\end{tabular}

\section{CI-level}

The condition indices (CI) yielded by fuzzy inference regarding the extent parameter $\mathrm{D}$ and the impact parameter $\mathrm{R}$ were divided into CI-levels by conducting reference conversion. The CI-levels were defined as follows: which is classified into 3 levels and transferred from CI value. Show the current state of the single structure. Subsequently, statistical concepts were applied and all CI-level values were calculated using the arithmetic mean method. Finally, the results were divided into the following three reference intervals: Interval 1 ranged from 0.75 to 1 (Level A), Interval 2 ranged from 0.74 to 0.45 (Level B), and Interval 3 ranged from 0.44 to 0 (Level C). A high score indicated a high level and increased satisfactory functions.

\section{Overall structural function index}

The differences between the OSFI and the CI-levels is that the CI-levels were developed based on inspector scores regarding the conditions of individual structures, and subsequently the current functions of individual structures were considered, whereas the OSFI involved overall considerations. In particular, all structures located in an inspection interval were included in the inspection, and the CIs of all structures were calculated using equations; the current overall $\mathrm{CI}$ of the interval served as a reference. OSFI denotes the functional degree of the entire area to calculate $\mathrm{U}$.

The OSFI in Equation (2) is a preliminary OSFI, which is the mean of the CIs obtained in the inspections of various cases. $\mathrm{N}$ represents the total number of inspected facilities.

$$
\text { OSFI }=\left(\frac{\sum \mathrm{C} 1}{\mathrm{n}}\right) * 10
$$

\section{E. Parameter U maintenance urgency}

At this stage, a questionnaire was used to determine the type of urgency level indicated by various OSFI values. This questionnaire was designed based on the analysis results of the previous questionnaire. In addition, the OSFI was divided into four levels. To investigate which urgent maintenance management strategies to use at various levels, the questionnaire included four maintenance urgency levels and was administered to 18 experts with an average experience exceeding 10 years.

The CV value was required to be less than or equal to 0.5 as the standard for determining whether the opinions of the experts were consistent. A CV value exceeding 0.54 indicates that the experts had inconsistent opinions, and such question items were excluded. Instead, the highest mean value was selected as the result (Table 4).
Table 4 Parameter U maintenance urgency questionnaire results table

\begin{tabular}{|c|c|c|c|c|c|}
\hline $\mathrm{U}$ & OSFI & Mean & SD & $\mathrm{CV}$ & \\
\hline \multirow{4}{*}{1} & $0 \leqq$ OSFI $<2.5$ & 5.00 & 0.70 & 0.14 & $*$ \\
\hline & $2.5 \leqq \mathrm{OSFI}<5$ & 4.13 & 0.69 & 0.17 & \\
\hline & $5 \leqq \mathrm{OSFI}<7.5$ & 0.00 & 0.00 & 0.00 & \\
\hline & OSFI $7.5 \leqq$ & 0.19 & 0.71 & 3.77 & \\
\hline \multirow{4}{*}{2} & $0 \leqq \mathrm{OSFI}<2.5$ & 3.94 & 0.51 & 0.13 & \\
\hline & $2.5 \leqq$ OSFI $<5$ & 5.13 & 0.62 & 0.12 & $*$ \\
\hline & $5 \leqq \mathrm{OSFI}<7.5$ & 0.81 & 1.32 & 1.62 & \\
\hline & OSFI $7.5 \leqq$ & 0.75 & 1.37 & 1.83 & \\
\hline \multirow{4}{*}{3} & $0 \leqq \mathrm{OSFI}<2.5$ & 0.00 & 0.00 & 0.00 & \\
\hline & $2.5 \leqq \mathrm{OSFI}<5$ & 3.25 & 0.76 & 0.23 & \\
\hline & $5 \leqq \mathrm{OSFI}<7.5$ & 4.31 & 0.62 & 0.14 & $*$ \\
\hline & OSFI $7.5 \leqq$ & 3.94 & 0.51 & 0.13 & \\
\hline \multirow{4}{*}{4} & $0 \leqq$ OSFI $<2.5$ & 0.94 & 1.15 & 1.23 & \\
\hline & $2.5 \leqq \mathrm{OSFI}<5$ & 4.50 & 0.69 & 0.15 & \\
\hline & $5 \leqq \mathrm{OSFI}<7.5$ & 4.25 & 0.73 & 0.17 & \\
\hline & OSFI $7.5 \leqq$ & 4.81 & 0.67 & 0.14 & $*$ \\
\hline
\end{tabular}

1) Mudslide-prone regions

Constant monitoring and observation should be performed to identify the disaster types in a region. Facilities in regions susceptible to mudslides should not be rebuilt or restored immediately.

The reference values yielded by the calculations can be converted to the defined parameter $U$ values. Subsequently, the corresponding intervals were imported. "Routine checks" apply to soil and water conservation facilities that have defects but require no immediate repair, and can thus be considered normal. "Follow-up checks" apply to facilities that exhibit apparent damage or require functional improvements or repairs as indicated by the assessments. "Attention to improvement" applies to facilities that exhibit local damage and require that repairs within 3 years to prevent the damage from deteriorating. "Immediate improvement" applies to facilities that are damaged and dysfunctional, necessitating repair within 1 year, as shown in Table 5 .

Table 5 OSFI and U- scale conversion table

\begin{tabular}{c|c|c}
\hline OSFI grading & U-value & Urgent recommendation \\
\hline $0 \leqq$ OSFI $<2.5$ & $\mathbf{1}$ & Routine inspection \\
$2.5 \leqq \mathrm{OSFI}<5$ & $\mathbf{2}$ & Tracking improvement \\
$5 \leqq \mathrm{OSFI}<7.5$ & $\mathbf{3}$ & Monitoring and improvement \\
$7.5 \leqq \mathrm{OSFI}$ & $\mathbf{4}$ & Immediate improvement \\
$\mathrm{DFD}$ & $\mathbf{5}$ & Monitoring observation \\
\hline
\end{tabular}

\section{EMPIRICAL CASE}

In the cases examined in this study, the maximum and minimum values of parameters $\mathrm{D}$ and $\mathrm{R}$ were selected from the intervals yielded by actual inspections to conduct combination inference. The inference results are shown in Table 6. Subsequently, the CI-levels and urgency values were calculated in both cases to offer recommendations for future maintenance. Brief explanations regarding the 
results for the two cases are provided in the following sections.

\section{A. Case Study}

The inspected structures are two groundsill works, the images of which are shown in Figures 1-1 and 1-2. Extensive erosion occurred in the groundsill works because of constant water scour.

The results of onsite assessments showed the scores that inspectors assigned for parameter $\mathrm{D}$ regarding Groundsill Works 1 was between 0.6 and 0.7 , and the scores for parameter $\mathrm{R}$ were generally between 0.3 and 0.5 because the effects were frequently considered minor. Regarding Groundsill Works 2, unilateral erosion occurred because of flow offset. Because of the smaller area, the scores of parameter D assigned by the inspectors during onsite inspections were between 0.2 and 0.3 , and the scores for parameter $\mathrm{R}$ were between 0.1 and 0.2 because the inspectors considered the effects to be minor.

\section{B. Inspection results and inference results}

In Case A, the OSFI, the urgency parameter U, and fuzzy inference result CI were substituted into Equation (2), yielding an OSFI of 3.39. The overall urgency $U$ corresponding to this value was identified in the interval to understand the level of urgency that the case necessitated. According to the analysis, 3.41 was in the 2 $\leqq$ OSFI $<5$ range, indicating that the structure was apparently damaged. Although the structural safety and functions remained unaffected, inspections should be conducted with increased frequently. In addition, the score for parameter $U$ was 2; therefore, follow-improvement suffices.

\begin{tabular}{|c|c|c|c|c|}
\hline \multicolumn{5}{|c|}{ Figure 1-1. Groundsill work NO.1 } \\
\hline tio & sults & & & \\
\hline D & $\mathbf{R}$ & $\mathrm{Ci}$ & $\begin{array}{l}\text { CI - } \\
\text { level }\end{array}$ & $\mathbf{U}$ \\
\hline 0.6 & 0.3 & 0.467 & $\mathrm{~B}$ & \multirow{4}{*}{2} \\
\hline 0.7 & 0.5 & 0.5 & $\mathrm{~B}$ & \\
\hline 0.2 & 0.1 & 0.178 & $\mathrm{~A}$ & \\
\hline 0.3 & 0.2 & 0.209 & A & \\
\hline 1 & 0.9 & 0.837 & $\mathrm{C}$ & \multirow{4}{*}{5} \\
\hline 1 & 1 & 0.837 & $\mathrm{C}$ & \\
\hline 0.8 & 0.7 & 0.806 & $\mathrm{C}$ & \\
\hline 0.9 & 0.8 & 0.836 & $\mathrm{C}$ & \\
\hline
\end{tabular}

\section{Discussion and analysis}

The inspection reports constituted individual assessments regarding the extent of damage (parameter D) and effects (parameter R) on the damaged structures. This study used fuzzy sets to integrate relevant semantics and scoring criteria and divided the structures into various CI-levels to reduce the substantial differences in scores resulting from subjectivity. Subsequently, the CI-levels, CIs, and OSFIs were used to obtain parameter $U$, which could serve as a maintenance timing reference.

Integrating visual inspections with fuzzy inference provides the following advantages:

1) Simplifying inspection procedures

The visual inspection assessments are limited to the damaged parts of structures, and undamaged components remain unexamined. Therefore, the inspection procedures are simplified, and the priorities in repairing damaged soil and water conservation facilities are assessed by using the CI-levels.

2) Modifying the damage inspection scoring system

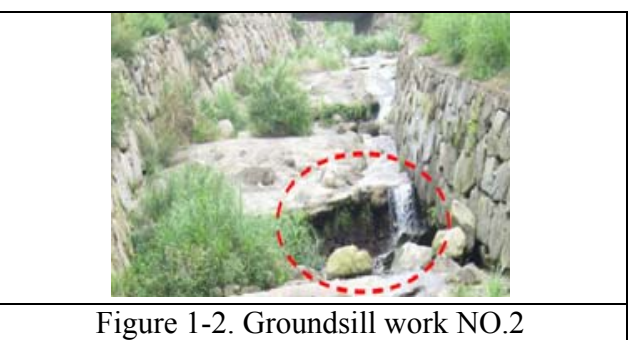

Fuzzy tools were employed in this study to reduce the difference in the assessments resulting from subjectivity. Following fuzzy inference and defuzzification, the CI was converted to the current CIlevel of a structure, thereby enhancing the consistency of the inspection results.

3) Understanding regional urgency using comprehensive condition values

Following inference and fuzzification, the individual CIs of the facilities could be understood clearly and the means of the overall conditions indicated the overall urgency. Recommendations regarding maintenance could be provided based on the $U$ value converted from the OSFI.

The results of this study can be used to effectively reduce the effects of subjectivity and calculate the functional intervals of structures and the overall urgency values of particular regions for facilitating maintenance repairs.

\section{CONCLUSION AND RECOMMENDATIONS}

The method developed in this study enables applying visual assessments to inspections regarding soil and water conservation facilities and concurrently reducing the inspection times, thereby increasing the number of routine inspections of soil and water conservation units performed annually. Fuzzy inference can be used to reduce inconsistencies in assessment criteria and assessment subjectivity effectively.

Evaluations of two cases showed that the application of fuzzy inference resolved the problems resulting from visual inspection and reduced subjective assessments, 
which result from personal differences. Subsequently, the CIs yielded by the fuzzy inference method can be used to evaluate the structural CI-levels further. The CIs of all structures were used to calculate the OSFIs, yielding the $U$ values, which indicated the urgency level. The $U$ value can enhance the accuracy of visual inspections and serve as a reference for achieving effective maintenance management. The proposed methods will enable the results to become accessible immediately following the inspections and serve as part of the facility archives of the regions.

\section{ACKNOWLEDGMENT}

Thanks for the Soil and Water Conservation Board Slopeland Conservation and Utilization group provide resources for research funding, and get relevant results.

\section{REFERENCES}

[1] H.T. Liu, and W. K. Wang, "An integrated fuzzy approach for provider evaluation and selection in third-party logistics," Expert Systems with Applications, vol. 36, No.3, 2009, pp.4387-4398. http://dx.doi.org/10.1016/j.eswa.2008.05.030.

[2] A. Poveda, and A. R. Fayek, "Predicting and Evaluating Construction Trades Foremen Performance: Fuzzy Logic Approach," Journal of Construction Engineering \& Management, Vol.135, No.9, 2009, pp. 920-929. http://dx.doi.org/10.1061/(ASCE)CO.1943-7862.0000061

[3] T. V. Reshmidevi, T. I. Eldho, and R. Jana, "A GIS-integrated fuzzy rule-based inference system for land suitability evaluation in agricultural watersheds", Agricultural Systems, Vol. 101, No.1-2, 2009, pp. 101-109. http://dx.doi.org/10.1016/j.agsy.2009.04.001

[4] i. Saleh, and S. i. Kim, "A fuzzy system for evaluating students' learning achievement", Expert Systems with Applications, Vol.36,
No.3,
2009
pp.
6236-6243 http://dx.doi.org/10.1016/j.eswa.2008.07.088

[5] N. Rezaeiniya, H. S. Zolfani, and E. K. Zavadskas, "Greenhouse locating based on ANP-COPRAS-G methods - an empirical study based on Iran", International Journal of Strategic Property Management, Vol.16, No.2, 2012, pp.188-200. DOI: $10.3846 / 1648715 X .2012 .686459$ 